\title{
Validity of the use of Schwartz formula against creatinine clearance in the assessment of renal functions in children
}

\author{
*H W Dilanthi ${ }^{1}$, G A M Kularatnam ${ }^{1}$, S Jayasena ${ }^{1}$, E Jasinge ${ }^{1}$, D B D L Samaranayake S $^{2}$ \\ V P Wickramasinghe ${ }^{3}$
}

Sri Lanka Journal of Child Health, 2017; 46(2): 155-159

\begin{abstract}
Introduction: Glomerular filtration rate (GFR) is used as a measure of overall renal function. Creatinine clearance $(\mathrm{CrCl})$ based estimation of GFR requires a 24-hour collection of urine which is cumbersome and time consuming. Instead, Schwartz formula is used for the estimation of GFR based on calculations. It is an estimate of GFR (eGFR) in children, derived from body length (L, in $\mathrm{cm})$, serum creatinine (Scr, in $\mathrm{mg} / \mathrm{dL}$ ) and a constant $(\mathrm{k}=0.55):$ GFR $=(0.55 \times \mathrm{L}) / \mathrm{Scr}$. However, it is important to cross validate this equation before using it in Sri Lankan children.
\end{abstract}

Objective: To cross validate the use of Schwartz formula against $\mathrm{CrCl}$, in a paediatric population in determining the glomerular function.

Method: A cross-sectional descriptive study was carried out at Lady Ridgeway Hospital for Children, Colombo, Sri Lanka. Forty seven children with chronic kidney disease from medical wards and nephrology clinic and 26 healthy children, between the ages of 3-12 years were recruited. In these patients GFR was determined using $\mathrm{CrCl}$ and calculated GFR by Schwartz formula using serum creatinine measure. The $\mathrm{CrCl}$ values were compared against eGFR derived from Schwartz formula. Correlation and limits of agreement between the two methods were evaluated.

Results: Although a weak but statistically significant correlation $(\mathrm{r}=0.476, \mathrm{P}<0.001)$ was observed between GFR determined by $\mathrm{CrCl}$ and that estimated by Schwartz formula in the whole

${ }^{1}$ Department of Chemical Pathology, Lady Ridgeway Hospital for Children, Sri Lanka, ${ }^{2}$ Department of Community Medicine, Faculty of Medicine, University of Colombo, Sri Lanka, ${ }^{3}$ Department of Paediatrics, Faculty of Medicine, University of Colombo, Sri Lanka

*Correspondence: dilanthiw@gmail.com

(Received on 31 July 2016: Accepted after revision on 04 October 2016)

The authors declare that there are no conflicts of interest

Personal funding was used for the project.

Open Access Article published under the Creative

Commons Attribution CC-BY CC License. population, Bland Altman plot showed that the two tests had acceptable limits of agreement in lower GFR values but the limits became wider at higher GFR values.

Conclusions: A weak but statistically significant correlation was seen between eGFR based on $\mathrm{CrCl}$ and eGFR derived from Schwartz formula.

DOI: http://dx.doi.org/10.4038/sljch.v46i2.8273

(Key words: Glomerular function, glomerular filtration rate, serum creatinine, creatinine clearance, Schwartz formula)

\section{Introduction}

Glomerular filtration rate (GFR) is a measure of the overall renal function in health and disease ${ }^{1}$. It is useful in determining treatment, monitoring disease progression, predicting the point at which renal replacement therapy will be required and providing a guide to adjust dosages of drugs excreted by the kidney in order to prevent toxicity ${ }^{1}$. Renal inulin clearance, considered as the gold standard for determining GFR, cannot be used routinely as it is time consuming and expensive ${ }^{1}$. Creatinine clearance $(\mathrm{CrCl})$ based estimations of GFR are commonly used due to their practicability in day to day clinical practice. However, they require a 24hour urine collection which is cumbersome and time consuming both for the patient and the staff.

Efforts have been made to improve rapid estimation of GFR for children using equations, without a 24hour urine collection. One such equation is the Schwartz formula, based on a mathematical relationship between serum creatinine (Scr) and GFR. The equation consists of the concept of height, as a surrogate measure of muscle mass, divided by Scr as a marker of glomerular function. Therefore, $\mathrm{eGFR}=\mathrm{k} \times \mathrm{L} / \mathrm{Scr}$, where eGFR represents estimated GFR in $\mathrm{ml} / \mathrm{min} / 1.73 \mathrm{~m}^{2}$, L represents height in $\mathrm{cm}$, Scr represents serum creatinine in $\mathrm{mg} / \mathrm{dL}^{3}$ and $\mathrm{k}$ is an empirical constant determined by comparing the L/Scr against measured $\mathrm{GFR}^{2,3}$. Schwartz et al revealed excellent agreement of eGFR using Schwartz formula with GFR estimated by $\mathrm{CrCl}(\mathrm{r}=0.935)$ or inulin clearance $(\mathrm{r}=0.905)^{3}$.

Limitations to the use of Schwartz formula have been noted. The constant $\mathrm{k}$ varies with the age and 
sex of the child ${ }^{2}$. The larger value for $\mathrm{k}(0.7)$ for adolescent boys indicates the greater rate of urinary creatinine excretion (depending on muscle mass) in this group of children ${ }^{2}$. Mouin et al reported that the eGFR calculated using Schwartz formula overestimates the GFR in patients with renal disease ${ }^{4}$.

\section{Objective}

To cross validate the use of Schwartz formula against $\mathrm{CrCl}$ as the criterion standard, in determining the glomerular function in Sri Lankan children.

\section{Method}

Children with kidney disease (chronic renal failure or nephrotic syndrome) between the ages of 3 to 12 years, who were admitted to medical wards or attended the nephrology clinic at Lady Ridgeway Hospital for Children, Colombo were recruited. Children with no past history of urinary tract infections, renal disease or abnormal urine analysis results and with a negative family history of renal disease were recruited as healthy children. Informed written consent was obtained from the parents and a random urine sample was obtained for microscopic (urinary full report) and biochemical evaluation (dipstick testing for urine protein).

Patients with urinary tract infections, haematuria, proteinuria and who had taken cephalosporins within the last 2 weeks were excluded. During the first interview demographic data including age, sex, body weight and height were obtained. Body weight was measured to the nearest $0.1 \mathrm{~kg}$ by a standard beam balance and height was measured to the nearest $0.5 \mathrm{~cm}$ without shoes using a wall-mounted stadiometer. A 24-hour urine collection was obtained along with a sample of blood drawn during the same 24 hour from each study subject. All parents were given clear instructions regarding collection of urine during the 24-hour period. Adequacy of the 24-hour urine collection was assessed by comparing the amount of total creatinine in the sample to the established realistic creatinine excretion rate in children $(70-195 \mu \mathrm{mol} / \mathrm{kg} /$ day). Any outliers were excluded from the study. All samples were analysed on the same day of collection upon receipt at the Department of Chemical Pathology, Lady Ridgeway Hospital.

Urine and serum creatinine were measured by modified rate Jaffe method. Between-run coefficients of variation for urine creatinine were $5.8 \%$ and $4.7 \%$ at $3004 \mu \mathrm{mol} / \mathrm{L}$ and $11,200 \mu \mathrm{mol} / \mathrm{L}$ levels of concentrations respectively. The between run coefficients of variation for serum creatinine were $6.1 \%$ and $4.2 \%$ at serum concentrations of 55 and $110 \mu \mathrm{mol} / \mathrm{L}$ respectively. All were analysed by Konelab Prime 30 fully automated analyser (Thermo Scientific, Finland). With each analytical run, quality control samples at 2 levels (high and low) were analysed. $\mathrm{CrCl}$ was expressed as $\mathrm{ml} / \mathrm{minute} / 1.73 \mathrm{~m}^{2}$ and eGFR was calculated using the Schwartz formula; $(0.55 \times \mathrm{L}) / \mathrm{Scr}$. The study protocol was reviewed and approved by the ethics review committee of Lady Ridgeway Hospital.

Data were analysed using SPSS statistical package. Standard descriptive methods (mean, median, standard deviation etc.) were used to describe the measured parameters. Correlation coefficients were used to quantify the association between the estimated GFR values. The limits of agreement between the two parameters were analysed by the Bland Altman plot.

\section{Results}

Twenty six healthy children and forty seven children with kidney disease were enrolled in the study. Demographic and anthropometric data of these children are given in table 1 .

Table 1: Demographic and anthropometric data for healthy children and children with kidney disease

\begin{tabular}{|l|c|c|}
\hline & Healthy children $(\boldsymbol{n = 2 6 )}$ & Children with kidney disease $(\boldsymbol{n}=\mathbf{4 7})$ \\
\hline Sex (girls/boys) & $17 / 9$ & $29 / 18$ \\
\hline Age (years) & $9.0(7.2-10.8)$ & $6.8(5.6-8.6)$ \\
\hline Height $(\mathrm{cm})$ & $133.5(119.9-143)$ & $114.0(105-129)$ \\
\hline Weight $(\mathrm{kg})$ & $27.5(22.9-36.5)$ & $22.0(16.5-6.5)$ \\
\hline BMI $\left(\mathrm{kg} / \mathrm{m}^{2}\right)$ & $15.6(14.5-19.5)$ & $15.4(14.1-17.4)$ \\
\hline
\end{tabular}

*Data expressed as median (range)

The difference in median weight and height between patients with kidney disease and normal healthy children may be due to the difference in age distribution in these two groups.
Biochemical parameters, $\mathrm{Scr}, \mathrm{CrCl}$ and eGFR using Schwartz formula for the healthy subjects and patients with renal disease are given in table 2 . 
Validity of the use of Schwartz formula against .... Sri Lanka Journal of Child Health, 2017; 46(2): 155-159

Table 2: Glomerular filtration assessment data of healthy children and children with kidney disease

\begin{tabular}{|l|c|c|}
\hline & Healthy children $(\boldsymbol{n}=\mathbf{2 6})$ & Children with kidney disease $(\boldsymbol{n}=\mathbf{4 7})$ \\
\hline Serum creatinine $(\mu \mathrm{mol} / \mathrm{L})$ & $51.5(45-55.25)$ & $39(36-50)$ \\
\hline Creatinine clearance $\left(\mathrm{ml} / \mathrm{min} / 1.73 \mathrm{~m}^{2}\right)$ & $93(78.7-109)$ & $92(75-110)$ \\
\hline e GFR $\left(\mathrm{ml} / \mathrm{min} / 1.73 \mathrm{~m}^{2}\right)$ & $124(112.25-145.5)$ & $140(119-155)$ \\
\hline
\end{tabular}

*Data expressed as median (range)

Due to the low number of patients with chronic renal failure and relatively higher number of patients with nephrotic syndrome recruited to the study, the Scr and $\mathrm{CrCl}$ values in the patients with kidney disease were within the normal range. A significant positive correlation between $\mathrm{CrCl}$ and eGFR ( $\mathrm{r}=0.476$, $\mathrm{P}<0.001$ ) was noted (Figure 1). According to Bland Altman plot, the limits of agreement were -20.7 to +96.1. (Figure 2).

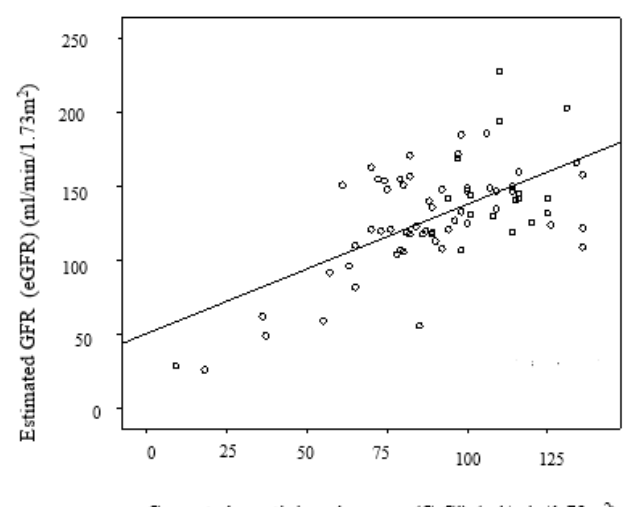

Figurel: Correlation between estimated glomerular filtration rate (eGFR) and creatinine clearance $(\mathrm{CrCl})$

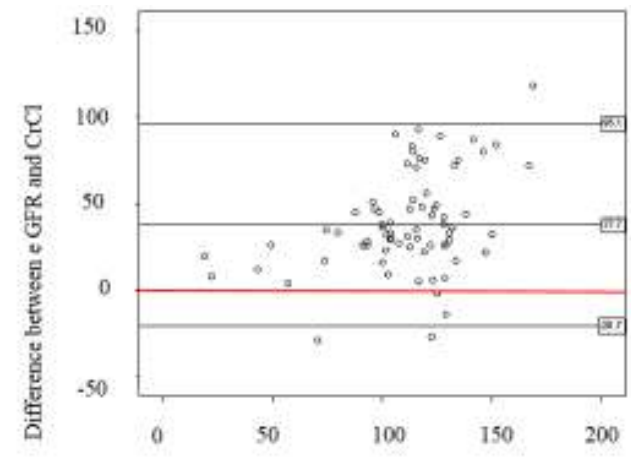

Average of $\mathrm{CrCl}$ and eGFR (mlminute/1.73 $\mathrm{m}^{2}$ ) Figure 2: Comparison between creatinine clearance $(\mathrm{CrCl})$ and estimated glomerular filtration rate (eGFR) using Bland Altman plot. The upper and lower lines indicate the $95 \%$ distribution of the population.

The limits of agreement became wider as the GFR value increased. Hence despite good correlation the agreement between eGFR and $\mathrm{CrCl}$ had wide limits of agreement especially at high levels of GFR. However almost all the values were within $\pm 2 \mathrm{SD}$ limits. According to the mean bias, eGFR overestimated the GFR assessed by $\mathrm{CrCl}$, by 37.7 $\mathrm{ml} / \mathrm{min} / 1.73 \mathrm{~m}^{2}$.

\section{Discussion}

Collection of 24-hour urine samples to calculate $\mathrm{CrCl}$ is cumbersome and prone to errors. In children, use of $\mathrm{CrCl}$ to determine $\mathrm{GFR}$ is unreliable as it requires toilet training and complete voiding. In addition in patients with chronic kidney disease extra renal clearance of creatinine would result in falsely high $\mathrm{CrCl}$ values. The ability of the Schwartz formula to estimate GFR accurately in children has been investigated broadly. The eGFR has been compared with the renal clearance of either an exogenous substance or endogenous creatinine. Studies have revealed that eGFR calculated by Schwartz formula overestimates the $\mathrm{CrCl}$ measured by renal clearance of substances ${ }^{4}$. Original Schwartz formula was developed in a group of patients with chronic kidney disease, using inulin clearance as the reference method and creatinine measured by a modified Jaffe reaction, which may have overestimated true creatinine value ${ }^{3}$. Many of the studies, which later evaluated the Schwartz formula in children, have substituted creatinine clearance for inulin clearance for the measurement of GFR, in assessing bias and precision of eGFR in different populations. Similarly we studied the validity of use of Schwartz formula in determining GFR in children by measuring Scr with the kinetic Jaffe method and $\mathrm{CrCl}$ as the reference method. Although the correlation coefficient is highly statistically significant, the strength of the correlation was low. According to the Bland Altman plot, it shows a wide difference between the values of measured and eGFR and this difference is widened in higher values of GFR. However there were only few patients with very low GFR values.

Hari et al based on the regression analysis, found the value of $\mathrm{k}$ to be 0.42 in Indian children. According to Hari et al creatinine was estimated by kinetic Jaffe method and clearance of 99mTc-DTPA was used as the reference method to measure GFR ${ }^{7}$. Applying this $\mathrm{k}$ value (0.42) instead of 0.55 , eGFR was recalculated using the data set of the current study. Then the data were re-analysed using correlation and Bland Altman plot. According to those results, the Bland Altman plot showed limits of agreement within -37.9 to +52.9 , somewhat low compared to the use of the original constant described by Schwartz, but still considerably wide and the 
agreement between eGFR and creatinine clearance showed wider limits of agreement especially at high levels of eGFR. However almost all the values were within \pm 2 SD limits. When the constant of the formula was changed to 0.42 , the mean bias reduced to $7.5 \mathrm{ml} / \mathrm{min} / 1.73 \mathrm{~m}^{2}$. Therefore, the use of a constant validated on an Indian population, improved the validity of estimation of GFR.

Mouin et al reported that the eGFR calculated using Schwartz formula overestimates the GFR in patients with renal disease ${ }^{4}$. At very low values of GFR $((\leq 15$ $\left.\mathrm{mL} / \mathrm{min} / 1.73 \mathrm{~m}^{2}\right)$, the percentage of overestimation of eGFR has been high $(164 \% \pm 42 \%)$, compared to the percentage overestimation of eGFR in children with normal $(10.3 \pm 3 \%)$ and mildly reduced GFRs $(90.3 \pm 14.3 \%)^{4}$. Therefore the degree of overestimation of GFR by Schwartz formula was inversely related to the level of renal function. Schwartz formula therefore would provide a better estimation for children with normal renal function or with mild renal insufficiency. However the reference standard used in this study was ${ }^{125}$ Iodineiothalamate clearance $(\mathrm{CIO})^{4}$.

Gbadegesin et al, in their study suggest that Schwartz formula should be validated for accuracy before routine use in clinical settings ${ }^{8}$. The study has been performed to assess the accuracy of the use of Schwartz formula in a group of healthy children and children with nephrotic syndrome. The results revealed that the eGFR overestimated the GFR measured by endogenous creatinine clearance ${ }^{8}$.

The current study is limited by its single-centre setting without the gold standard measure of GFR (e.g. iohexol, inulin clearance) and small number of patients with severe chronic kidney disease. Low muscle mass in undernourished children, may influence the value of $\mathrm{k}$, and may affect the GFR estimation. Thus, it may lead to over-estimation of GFR in this subset ${ }^{4,5}$. Moreover, the value of $\mathrm{k}$ should be different based on the method of estimation of Scr. Schwartz et al., using the enzymatic method of creatinine estimation in a population with chronic kidney disease, recently proposed a new $\mathrm{k}$ value of $0.413^{6}$. To improve the bias and accuracy of the GFR estimation, it is important for all the paediatricians to understand that the value of $\mathrm{k}$ should be locally derived based on the method of creatinine estimation, reference GFR estimation and the local population characteristics.

\section{Conclusions and recommendations}

In this study population, estimation of GFR by Schwartz equation did not agree with the GFR assessed by the criterion method. A significant bias was seen between eGFR and the GFR assessed by $\mathrm{CrCl}$. Although a weak, but statistically significant relationship existed between $\mathrm{CrCl}$ and eGFR by Schwartz formula, it could be improved by determining the most appropriate constant ' $\mathrm{k}$ ' value used in the formula. Therefore the $\mathrm{k}$ in the Schwartz formula may have to be validated to suit the population under study before routine use in clinical settings.

\section{Acknowledgements}

We acknowledge the medical laboratory technologists in the Department of Chemical Pathology, Lady Ridgeway Hospital for Children, Sri Lanka for their support in performing biochemical investigations.

\section{References}

1. Edmund L, David J, Newman, Christopher P, Price. Kidney Function Tests. In: Burtis CA, Ashwood ER, Bruns DE. Teitz Textbook of Clinical Chemistry and Molecular Diagnostics $4^{\text {th }}$ edition. USA: Elsevier Saunders; 2006: p.797-826.

2. Schwartz GJ, Brion LP, Spitzer A. The use of plasma creatinine concentration for estimating GFR in infants, children and adolescents. Pediatric Clinics of North America 1987; 34:571-90.

https://doi.org/10.1016/S00313955(16)36 251-4

3. Schwartz GJ, Haycock GB, Edelmann CM, Spitzer A. A simple estimate of glomerular filtration rate in children derived from body length and plasma creatinine. Pediatrics 1976; 58: 259-63.

PMid: 951142

4. Mouin G, Seikaly, Richard B, Geeta B, Billy S, Arant J. Limitations to body length/serum creatinine ratio as an estimate of glomerular filtration rate in children. Paediatric Nephrology 1996; 10(6): 70911.

https://doi.org/10.1007/s004670050195

5. Hari P, Bagga A, Mahajan P, Lakshmy R. Effect of malnutrition on serum creatinine and cystatin C levels. Pediatric Nephrology 2007; 22: 1757-61. https://doi.org/10.1007/s00467-007-0535$\mathrm{x}$

PMid: 17668246

6. Schwartz GJ, Muñoz A, Schneider MF, Mak RH, Kaskel F, Warady BA, et al. New equations to estimate GFR in children with 
CKD. Journal of the American Society of Nephrology 2009; 20: 629-37.

https://doi.org/10.1681/ASN.2008030287

PMid: 19158356 PMCid: PMC2653687

7. Hari P, Biswas B, Pandey R, Kalaivani M, Kumar R, Bagga A. Updated height- and creatinine-based equation and its validation for estimation of glomerular filtration rate in children from developing countries. Clinical and Experimental Nephrology 2012; 16: 697-705. https://doi.org/10.1007/s10157-012-06182

PMid: 22526484

8. Gbadegesin RA, Adeyemo AA, Asinobi $\mathrm{AO}$, Osinusi K. Inaccuracy of the Schwartz formula in estimating glomerular filtration rate in Nigerian children. Annals of Tropical Paediatrics 1997; 17(2): 179-85. https://doi.org/10.1080/02724936.1997.11 747884

PMid: 9230984 